Musées, Patrimoine et Culture scientifiques et techniques

$160 \mid 2015$

juillet-août 2015

\title{
Les impacts des écrans tactiles sur les visiteurs dans les musées
}

Charlotte Steiner et François H. Courvoisier

\section{(2) OpenEdition \\ Journals}

Édition électronique

URL : http://journals.openedition.org/ocim/1539

DOI : 10.4000/ocim.1539

ISSN : 2108-646X

Éditeur

OCIM

Édition imprimée

Date de publication : 1 juillet 2015

Pagination : 10-16

ISSN : 0994-1908

Référence électronique

Charlotte Steiner et François H. Courvoisier, « Les impacts des écrans tactiles sur les visiteurs dans les musées », La Lettre de I'OCIM [En ligne], 160 | 2015, mis en ligne le 01 juillet 2016, consulté le 01 mai 2019. URL : http://journals.openedition.org/ocim/1539; DOI : 10.4000/ocim.1539

Ce document a été généré automatiquement le 1 mai 2019.

Tous droits réservés 


\title{
Les impacts des écrans tactiles sur les visiteurs dans les musées
}

\author{
Charlotte Steiner et François H. Courvoisier
}

Détail du dispositif tactile "drags balls" (sphères lumineuses) dans l'exposition Univers de Particules présentée au CERN à Genève.

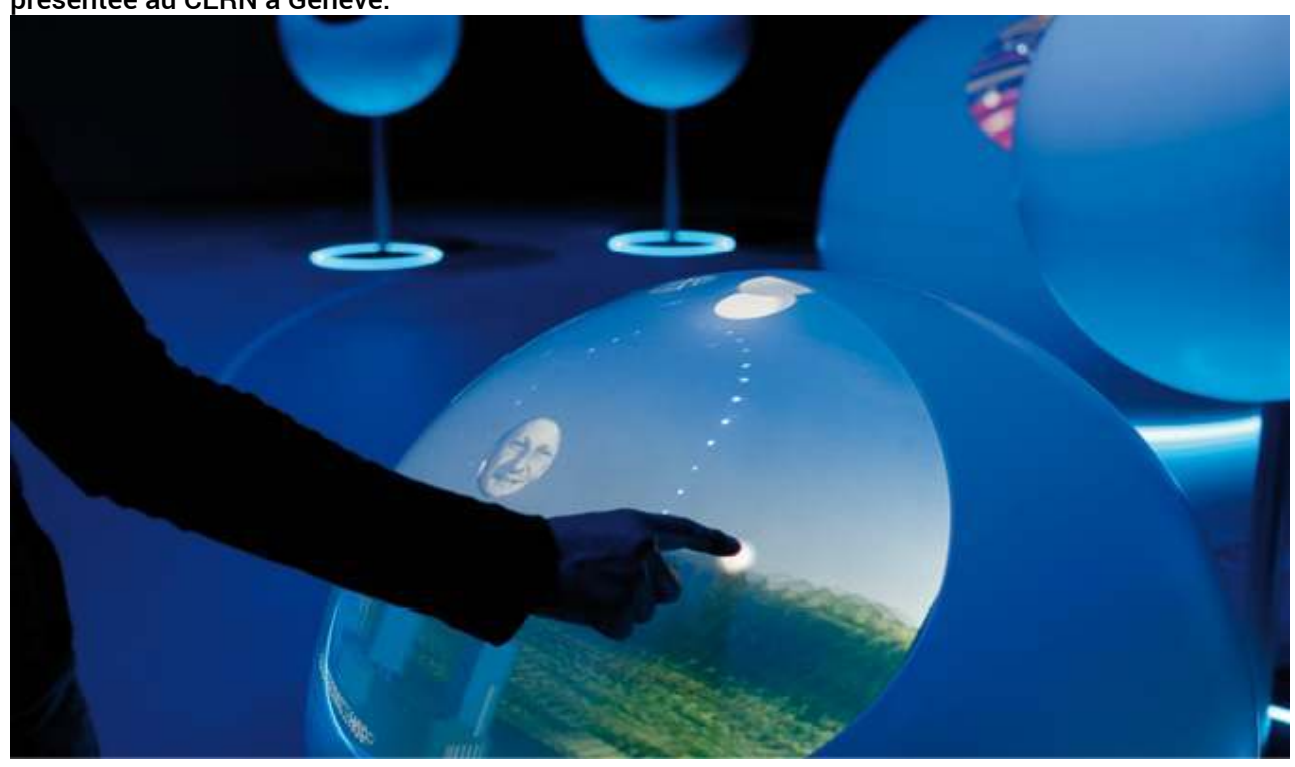

(c) CERN

1 La diversité et la multitude de musées, en ce début du XXI ${ }^{\mathrm{e}}$ siècle, sont un élément non négligeable dans le paysage culturel. De ce fait, les institutions muséales se font concurrence et doivent trouver leur place dans "l'industrie des loisirs". Afin de perdurer et d'exceller, elles doivent devenir attractives pour ne pas être relayées au rang de simples gardiennes du patrimoine.

2 Dans un environnement numérique de plus en plus démocratisé, de nombreux musées misent sur l'intégration des écrans tactiles sur bornes fixes, au sein même de leurs espaces d'exposition temporaires ou permanents. Auparavant utilisées en annexe de la 
visite en guise de renseignements ou comme guichets d'achat de billets, ces bornes deviennent autant un mobilier à part entière qu'un dispositif de médiation dans les salles d'expositions.

3 Les musées sont alors englobés dans cette nouvelle appréhension de la réalité, redéfinie par les dispositifs numériques et tactiles. Ceux-ci donnent naissance à une pratique novatrice, à une expérience du musée modernisée et contemporaine. Cette évolution muséale met en évidence une problématique capitale: la numérisation des objets de musées. Elle recompose l'approche et la compréhension des objets, le tout dans une scénographie redéfinie par les écrans tactiles. Tout ceci engendre de nouveaux comportements gestuels et visuels, ainsi que de nouvelles implications physiques et émotionnelles.

4 Il est alors capital de mieux cerner quel est le bénéfice émotionnel, l'attractivité nouvelle de ces dispositifs tactiles auprès des visiteurs de musées, car leur intégration pose plus que jamais la question de l'interface avec les publics au cours de la visite de l'institution. Pour cela, nous avons effectué une recherche de terrain, qui a été réalisée selon une méthodologie qualitative exploratoire au moyen d'un questionnaire semi-directif remis aux visiteurs après leur visite du musée : les enquêtes ainsi réalisées ne permettent pas de généraliser une réalité mais de proposer une tendance.

Chaque enquête a duré une semaine entre mars et juin 2014. Une cinquantaine de visiteurs ont été interrogés par musée. Pour ce faire, nous avons collaboré avec quatre musées : deux à Bordeaux, le musée des Beaux-Arts pour sa réouverture et le musée d'Aquitaine pour ses nouvelles salles sur Bordeaux port(e) du monde, 1800-1939; deux en Suisse : le musée Olympique à Lausanne pour sa réouverture récente puis, le Microcosm et Univers de particules, deux espaces muséaux du CERN (Centre européen de recherches nucléaires) à Meyrin.

6 Le but de notre étude est de comprendre ce que pensent vraiment les visiteurs devenus, en quelque sorte, les expérimentateurs de cette nouvelle médiation numérique que sont les écrans tactiles : comment les utilisent-ils ? Sont-ils enthousiastes ou réfractaires? Ces surfaces tactiles sont-elles si séduisantes qu'on nous le donne à penser? Et ne risquentelles pas de dénaturer le rapport du visiteur à l'œuvre vraie, authentique, originale?

\section{La réécriture du monde}

7 La numérisation des objets, sinon la dématérialisation de tous les originaux, "recompose toute l'approche et la compréhension du patrimoine" (Coutaz et Cosandier, p. 3). Walter Benjamin et Jean Baudrillard avaient déjà, à leur manière et dans leur contexte, amorcé la réflexion sur le hic et nunc de l'œuvre originale, son aura dans l'espace face à la reproductibilité technique grandissante.

8 Aujourd'hui, la réflexion est toujours ouverte : l'appellation "authentique" est préférée à celle d'"original" qui, selon Gilbert Coutaz et Jean-François Cosandier, ne fait plus sens devant la facilité de reproduction. Nous entrons dans une phase de séduction numérique que nous pourrions appeler "nouvelle réalité". Celle-ci offre une nouvelle perception de l'œuvre authentique. Lorsque nous feuilletons les images numérisées sur un écran tactile (ou sur Internet) avons-nous toujours conscience qu'il s'agit d'originaux numérisés? Réalisons-nous que le format numérisé ne correspond pas toujours au format original de 
l'œuvre? Il s'agit là d'un changement de perspective dans l'appréhension spatiale et visuelle de la réalité de l'œuvre.

Dès lors qu'une œuvre est numérisée, c'est au musée d'en choisir le format adéquat d'exposition. Mais ces écrans ne permettent pas de la visualiser telle qu'elle est réellement. Si les formats varient, c'est parce que les écrans tactiles permettent de compacter intelligemment les dimensions des œuvres et des documents. Est-ce à dire que l'original a perdu de son intérêt majeur? Non. Cependant, les reproductions ont un aspect démocratique et culturel évident. Elles n'existent que parce qu'il n'y a qu'un original.

La présentation animée du chemin de fer dans la salle Bordeaux port(e) du monde, 1800-1939 au musée d'Aquitaine.

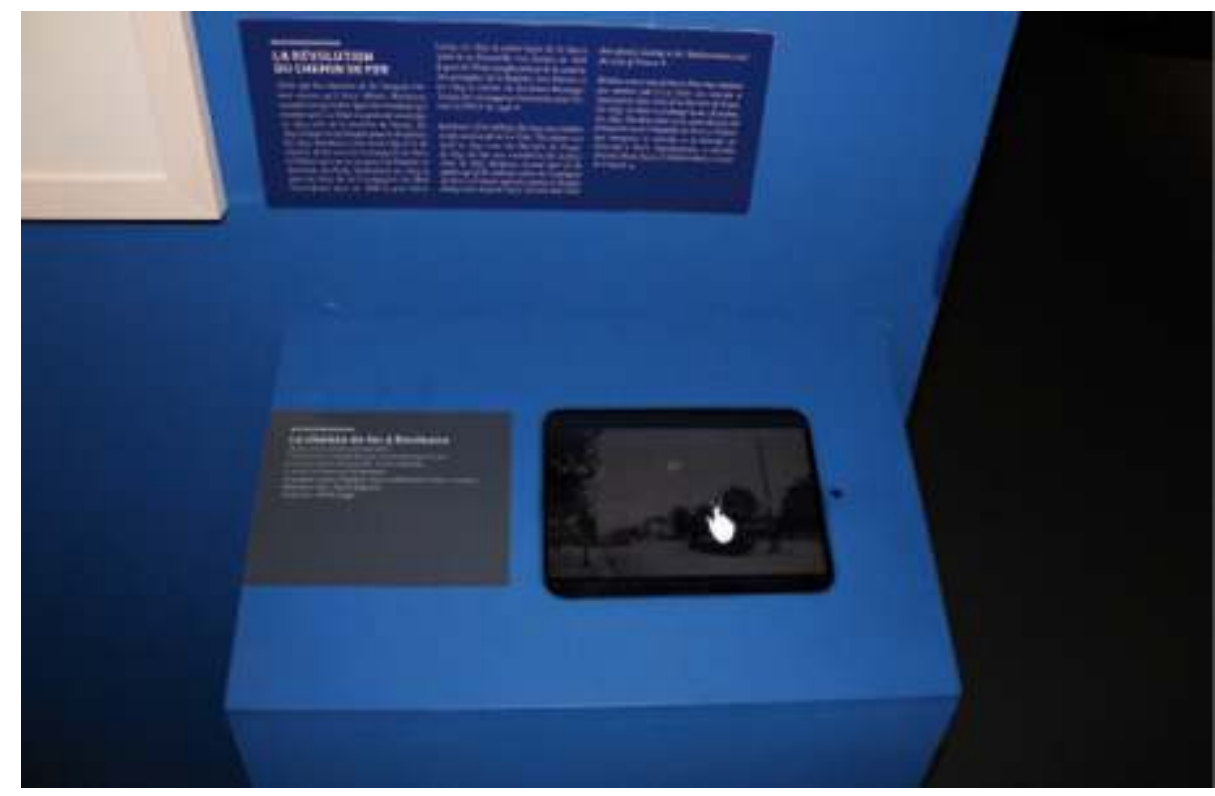

(C) Musée d'Aquitaine/Lysiane Gauthier

10 Aujourd'hui, "la multiplication des expérimentations numériques menées par les musées pour aider "[...] les publics à organiser les visites in situ se structure en une offre standard" (Torres, Treussard, Édouard-Baraud et D'Anglejan, p.13). Le CLIC (Club innovation et culture) France recense à cet effet, au 18 février 2015, 332 applications numériques dans les musées de France, mobiles ou non. Il devient normal, pour ne pas dire banal, d'utiliser et d'installer ces dispositifs tactiles dans l'espace d'exposition. Dans ce sens, citons les propos tenus lors du Forum d'Avignon en 2010 : les écrans multipoints sont en train de s'imposer comme les interfaces les plus ergonomiques pour permettre l'accès aux contenus (Torres, Treussard, Édouard-Baraud et D’Anglejan, p. 37).

11 Les conservateurs des musées interrogés lors de notre étude expriment qu'il s'agit d'une question de dosage entre les objets d'exposition réels et les dispositifs interactifs afin de ne pas dénaturer l'espace muséal. Ils rejoignent ce qu'exprime justement Mariève Bertrand : il "[...] faut rester vigilant dans l'utilisation [des technologies] afin d'éviter qu'elles ne supplantent les objectifs des dispositifs, [...]" (Bertrand, p. 5). Les propos d'Yves-Armel Martin abondent dans ce sens: "[...] on devra rechercher la simplicité, le dépouillement et le non empilement des médias" (Martin, p. 121). Ces affirmations répondent alors directement aux inquiétudes latentes: l'exposition elle-même ne peut-elle pas être dépassée par ces 
supports? Les dispositifs doivent susciter la curiosité non pas la rebuter, être "[...] envisagés comme des éléments complémentaires des ceuvres ou des thématiques" et ne doivent "[...] jamais camoufler les cuvres ni les rendre inaccessibles" (Balloffet, Courvoisier et Lagier, p. 12). En d'autres termes, "le musée ne doit pas ressembler à une FNAC", selon Geneviève DupuisSabron, conservatrice du XIX siècle au musée d'Aquitaine. C'est une réflexion qui a été soutenue dans les quatre musées dont il est question ce jour.

\section{Immersion dans la scénographie}

12 L'immersion muséale est immédiate dès le seuil de l'institution franchi. Une scénographie réussie est une médiation spatiale, capitale pour le message muséal. Chaque musée de notre échantillon opte pour des scénographies adaptées à ses contenus. De ce fait, ils n'exploitent pas les écrans tactiles de la même manière, ni avec la même importance.

Avant chaque réalisation des enquêtes auprès des publics, nous avons rencontré les conservateurs et/ou responsables de chacun des musées choisis pour ce travail. Il s'agissait de saisir le choix scénographique de chaque institution afin de pouvoir, ensuite, en discuter avec les visiteurs. Nous avons sélectionné des institutions de type différent afin de confronter des publics ayant des affinités sensiblement distinctes selon les musées qu'ils visitent.

14 Ainsi, la responsable des collections XIX ${ }^{e}$ siècle au musée d'Aquitaine, craignant que les dispositifs ne plaisent pas et qu'ils dérangent, souhaitant conserver le plus possible le format des archives (photographies et cartes postales) a opté, avec le scénographe, pour des tablettes iPad dans l'espace d'exposition. Elles sont dispersées au bord des salles, pour ne pas gêner la déambulation. Leur mise en valeur est discrète et parcimonieuse. Elles permettent "de gagner de la place et d'éviter une saturation visuelle" d'après les propos de la conservatrice. Ce procédé permet aux visiteurs de ne pas s'éparpiller sous la masse d'informations visuelles et se concentrer sur les expôts.

Le musée des Beaux-Arts de Bordeaux ${ }^{1}$, souffrant d'un manque de place évident, a choisi la même solution, des tablettes de type iPad afin de donner à voir davantage d'archives sonores, de documents et de tableaux numérisés. Serge Fernandez, documentaliste, s'en justifie : "la muséographie est déjà proche du mode d'exposition 'Touche-Touche' du XVIIIe siècle, nous devons, sans s'y opposer, s'en démarquer". Légèrement inclinées, sur des présentoirs neutres de couleur gris métallique, à hauteur des hanches, il s'agissait de disposer une douzaine de tablettes, deux sur chaque sujet en lien avec le contenu des salles pour ne pas gêner la déambulation. De ce fait, les dispositifs ont été placés en bord de salle voire dans les coins. 
Au musée Olympique de Lausanne, l'écran LED Multitouch permet la consultation à plusieurs comme dans la partie de l'exposition permanente Les Temps forts.

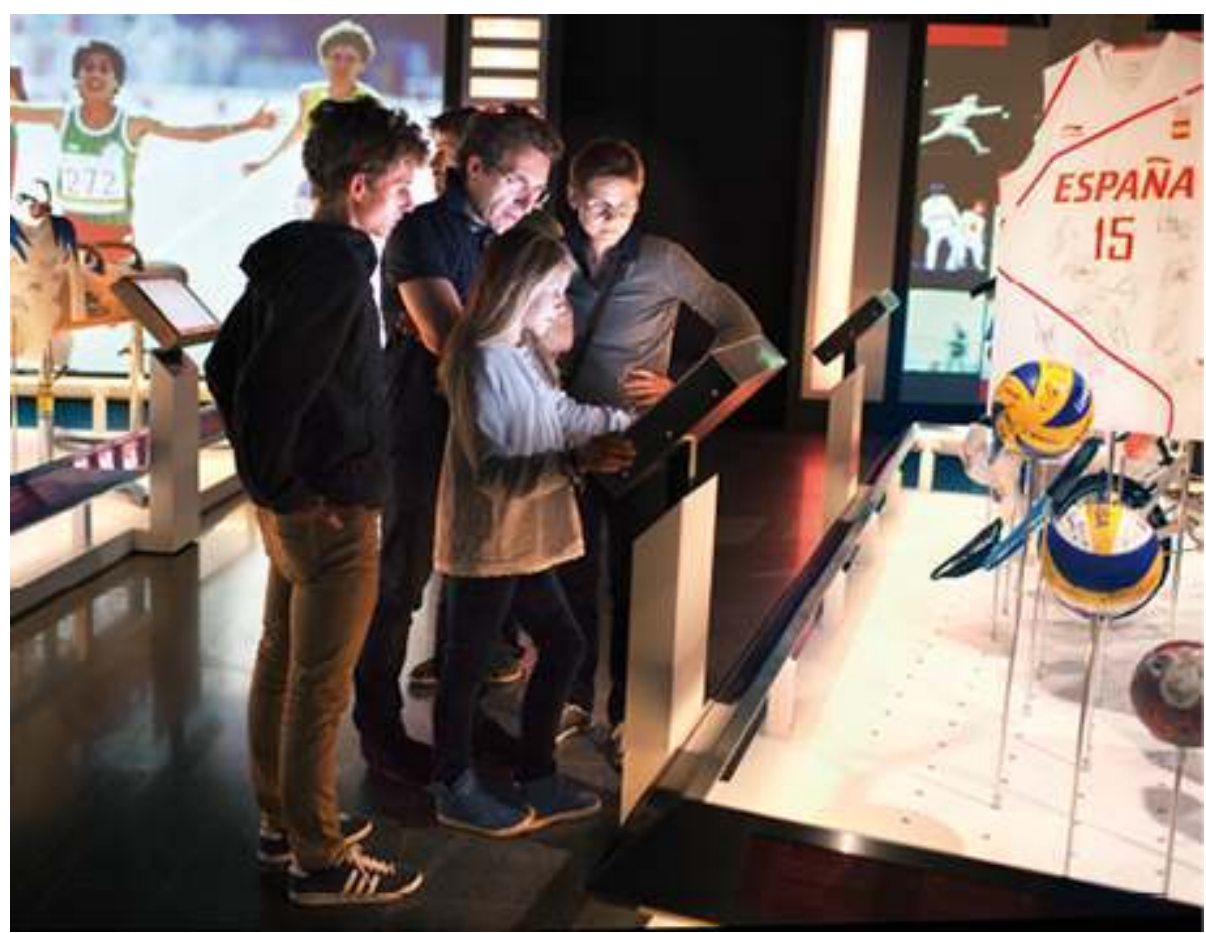

(c) ClO/Lydie Nesvadha

Le musée Olympique, suite aux travaux de mapping et de benchmarking, a choisi d'utiliser des écrans LED Multitouch créant une synergie avec l'architecture du bâtiment. Ces écrans fonctionnent comme des compléments avec les expôts. Inclinés, ils sont parsemés tout le long des trois étages de l'exposition permanente à hauteur d'adultes et sont présents dans chacune des salles. Ce procédé a permis d'exposer énormément d'archives photographiques et des vidéos. Les contenus sont alors étayés sans "alourdir la visite" selon les propos de Frédérique Jamolli, conservatrice.

Finalement, voici les choix du Microcosm et de Univers de particules au CERN. Le premier n'ayant actuellement pas encore fait 1\&apos;objet d'une rénovation, et suite aux remarques des visiteurs trouvant les propos de l'exposition trop difficiles d'accès et l'affichage sur panneau plutôt abrupt, des tablettes iPad y sont exposées pour délivrer des informations complémentaires dans l'exposition. Ce choix a été motivé "dans l'attente de la rénovation de ses lieux et pour expliciter les objets exposés", selon Bernard Pellequer, responsable de programme. À l'inverse, Univers de particules, aménagé depuis 2010 et basé sur le "wow factor", fait progresser le visiteur plongé dans une obscurité partielle parmi les particules de notre système solaire et l'histoire du Big Bang au travers de "drags balls" - sphères lumineuses -, dont les surfaces sont tactiles. Elles délivrent donc l'essentiel du contenu muséal. Ici, les tablettes sont purement informatives dans Microcosm alors que les écrans tactiles de l'Univers de Particules sont nécessaires pour saisir le discours muséal.

Plus largement, les écrans tactiles invitent donc à une forme de communication interdépendante avec l'humain, permettant ainsi l'accès aux contenus numérisés. Le visiteur est invité à les toucher dans l'espace muséal. Fini de déambuler les bras croisés derrière le dos. Il est davantage actif dans sa visite étant dans un rapport dynamique de 
captation du savoir. Il s'approprie plus immédiatement l'espace et participe à sa visite en se donnant lui-même la possibilité d'accéder à de l'information supplémentaire.

Auparavant, le visiteur était habitué à regarder l'œuvre originale, de manière sacrale, de face. Avec l'intégration des écrans tactiles sur bornes, la visualisation de l'objet numérisé se fait de manière inclinée ou à la verticale suivant la manière dont sont fixés les écrans. Le comportement visuel engendre, de fait, une nouvelle gestuelle. Le fait d'avoir à disposition ces écrans interactifs "[...] donne à voir la transformation plutôt que la contemplation" (Vol, p. 71). Ils offrent la possibilité d'une "[...] manipulation désacralisante des contenus muséaux" (Vidal, p. 73). La réflexion de l'original, de l'authentique, engendre directement celle de la manipulation de sa copie numérique, manipulation du réel et de ses métamorphoses.

Les écrans tactiles permettent de toucher le substitut numérique de l'œuvre unique car ils autorisent ce qui, jusqu'alors, était impossible: distordre les dimensions des œuvres. Effectivement, la possibilité de zoomer donne accès à des détails de l'œuvre, en gros plans. Cela redéfinit l'œuvre et lui donne sa "nouvelle réalité". Ces dispositifs interactifs permettent "[...] d'atteindre des réalités qu'ignore toute vision réelle [...]" (Benjamin, p. 14), l'accès à l'intimité de l'œuvre qui, à l'œil nu, passe inaperçue. Jusqu'alors, rien ne prouve définitivement que les visiteurs soient conquis par ces écrans tactiles. D'après Yves-Armel Martin, "[...] il est difficile de deviner comment les publics vont réagir et comprendre ce qu'il leur est proposé" (Martin, p. 126).

La partie de l'exposition Bordeaux port(e) du monde, 1800-1939 consacrée à l'industrie navale à Bordeaux propose différentes tailles d'écran.

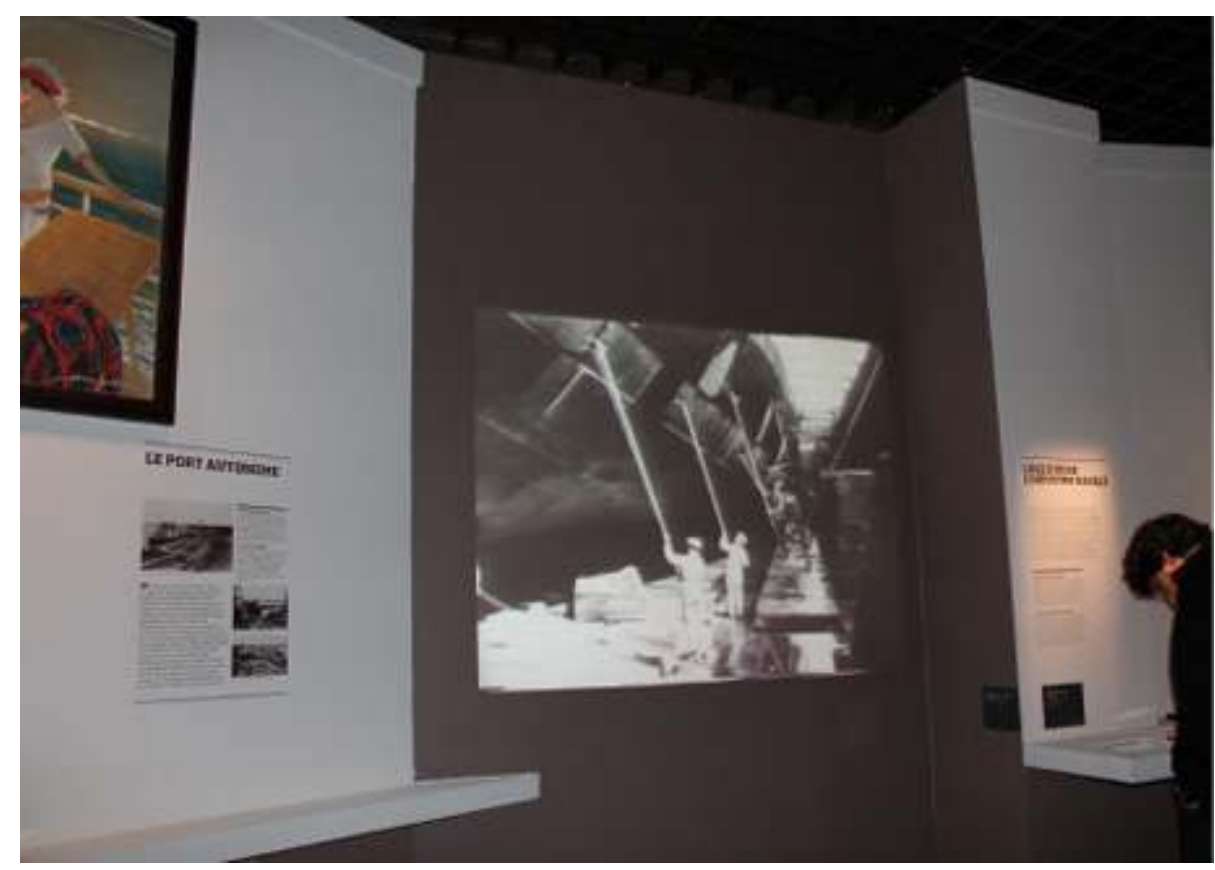

(c) Musée d'Aquitaine/Julie Guiroy

\section{La parole aux visiteurs}

Les questions fermées du questionnaire remis aux visiteurs comportaient des questions de type: "Êtes-vous déjà venu au musée? cochez oui ou non", "Avez-vous utilisé des écrans 
tactiles? cochez oui ou non", "Utilisez-vous dans votre vie quotidienne des écrans tactiles? cochez oui ou non". Puis, des questions, plus ouvertes comme "Que pensez-vous de la mise en valeur de ces écrans tactiles dans l'espace muséal (esthétique, ergonomie, situation dans l'espace) ?", " D'après vous, les nouvelles technologies peuvent-elles remplacer la visite guidée ? Et pourquoi ?".

Le choix des questions fermées permettait de pouvoir faire des tris généraux entre, par exemple, les utilisateurs et non utilisateurs et finalement, de faire connaissance avec le type de visiteurs qui remplissaient le questionnaire. Nous avons mis en valeur les questions ouvertes car elles laissaient le visiteur libre de noter ce qu'il souhaitait. Certains ont répondu à tous les aspects, d'autres, plus inspirés, se sont focalisés sur une problématique qui les tenait davantage à cœur de par leur formation, leurs intérêts personnels. Nous ne voulions pas diriger leurs réponses en leur proposant de donner une note de 1 à 6 (1 étant la moins bonne) mais plutôt nous intéresser linguistiquement aux termes qu'ils utilisaient. Ce dernier point, très important, nous a permis de créer notre tableau analytique. Les résultats ont alors été traités selon trois critères: la mise en valeur, les contenus puis l'ergonomie des écrans. Chaque enquête ayant été réalisée sur la durée d'une semaine environ avec au total 200 visiteurs, nous ne pouvons pas retenir des typologies précises de visiteurs. L'étude ne visait qu'à constituer un échantillon diversifié afin de pouvoir en tirer des tendances générales.

Globalement, il ressort que les visiteurs des musées interrogés ne venaient pas pour la première fois dans ces lieux. Leur présence était due soit à une exposition temporaire ou à la rénovation des salles d'exposition. Seul le CERN affiche un résultat de $94 \%$ de nouveaux visiteurs, ceci étant dû à des raisons davantage professionnelles, congrès, voyage d'études, voyage d'affaires... ce qui est directement lié aux provenances des visiteurs. Effectivement, le CERN avec une proportion de $96 \%$ et le musée Olympique avec 88 \% reçoivent davantage de visiteurs étrangers ; ils ont un rayonnement international, le CERN étant l'Organisation Européenne pour la Recherche Nucléaire et le musée Olympique proche du siège international du CIO (Comité international olympique). À l'inverse, les deux musées à Bordeaux ont des publics nationaux pour ne pas dire locaux.

Nous avons constaté que la majorité, pour ne pas dire la plupart des visiteurs (67\% au musée des Beaux-Arts, $82 \%$ au musée d'Aquitaine, $85 \%$ au musée Olympique et $90 \%$ au CERN), sont des utilisateurs au quotidien d'écrans tactiles. Les quelques non utilisateurs motivent leur réponse négative par le coût de ces objets, le manque d'intérêt et la difficulté d'utilisation. C'est une information capitale pour notre étude car cela nous permet, de prime abord, d'avoir affaire à un public plutôt averti.

\section{La mise en valeur}

Notre enquête révèle que la visibilité fait entièrement partie d'une intégration du numérique réussie ou non dans les espaces d'exposition. Le terme de discret a souvent été employé par les visiteurs, soit pour exprimer que la disposition des écrans ne dérange ni n'éclipse les autres canaux d'informations, soit de manière plus péjorative pour désigner le malaise des visiteurs car ils ne les ont pas aperçus durant leur visite. Au musée des Beaux-Arts, $30 \%$ des visiteurs seulement a utilisé les écrans tactiles, la plupart d'entre eux ne les ayant justement pas vus: "les gens passent devant sans les voir", "on ne les trouve pas facilement" ! 

étaient "peu voyantes", "pas assez indiquées". Le souci esthétique de ne pas vouloir imposer les écrans aux visiteurs, de ne pas les déranger dans leur visite en optant d'exposer les écrans en bord de salles ou de manière horizontale risque de les faire passer à côté de l'utilisation novatrice que sont ces dispositifs tactiles. Parfois même, le choix des supports sobres a été jugé de manière négative car il accentue cette non visibilité. Conjugués avec la mise en veille des écrans lors de leur non utilisation, cela n'a fait qu'accentuer la discrétion voire l'invisibilité des dispositifs. Mariève Bertrand l'exprime justement : "La difficulté de trouver un juste milieu dans l'introduction des technologies réside sans doute, pour partie, au niveau de la conception même de l'exposition" (Bertrand, p. 6).

Des dispositifs parfois trop discrets selon les visiteurs, comme dans la salle $\mathrm{XIX}^{\mathrm{e}}$ siècle du musée d'Aquitaine consacrée aux costumes.

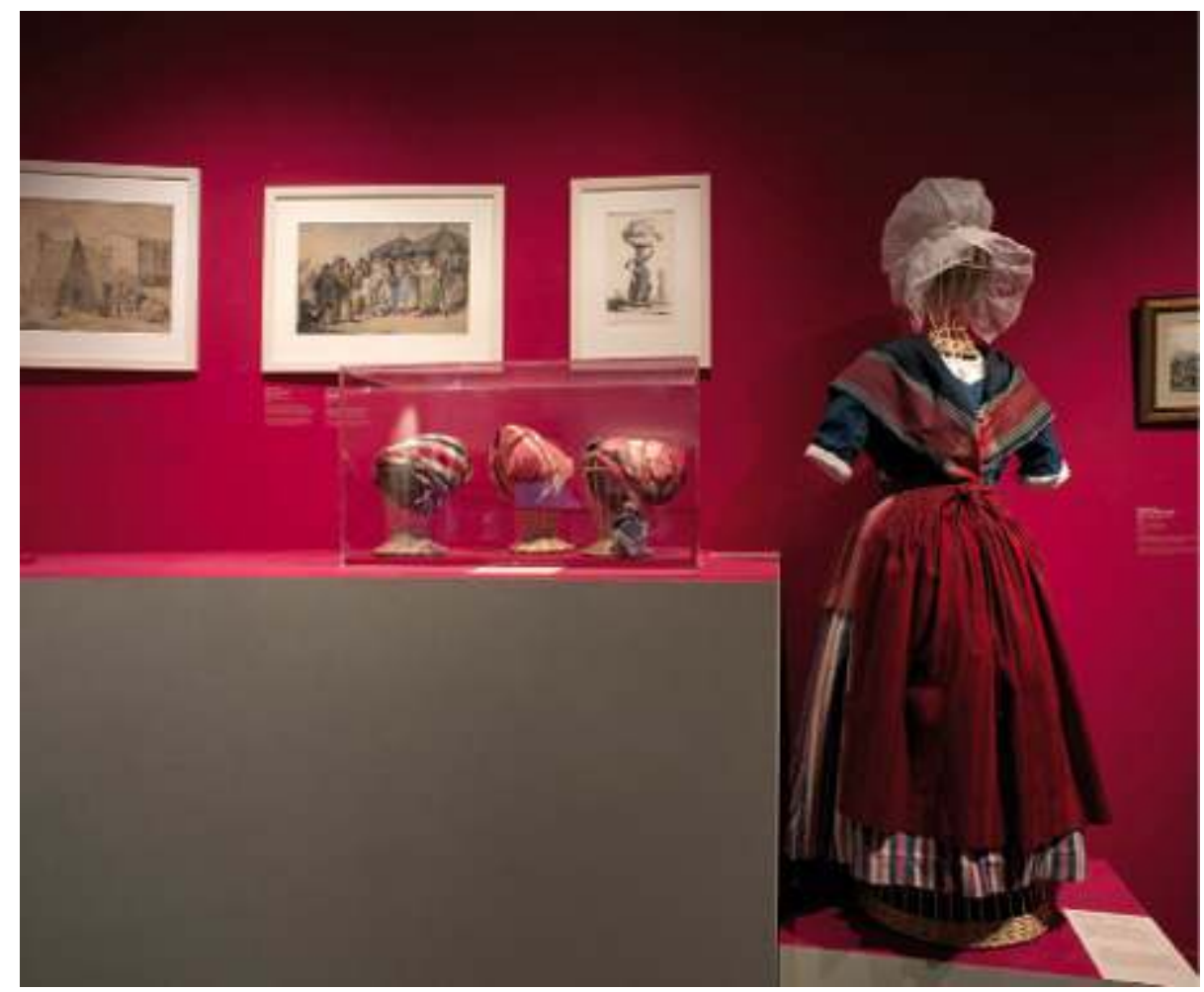

(C) Musée d'Aquitaine/Lysiane Gauthier

La taille des écrans joue aussi un rôle primordial dans l'appréciation de ceux-ci. Ils ont été jugé "trop petits" aux Beaux-Arts, "pas assez grands" au musée d'Aquitaine mais "agréables" au musée Olympique et "novateurs" dans Univers de Particules. Un petit écran de type iPad n'invite donc pas le public à être curieux, sa lisibilité étant moindre. Bertrand Pellequer le relevait justement en affirmant que le mode d'affichage est tout aussi important que le contenu que l'on souhaite mettre à disposition sur les écrans.

Cette problématique liée à la discrétion sous-tend celle de l'intégration visuelle des tablettes dans l'espace. Les visiteurs expriment "que les tablettes se fondent bien dans l'exposition, qu'elles trouvent leur place sans choquer". Dans le même ordre d'idées, "qu'elles n'éclipsent pas les autres canaux d'informations". D’un point de vue lexico-sémantique, le visiteur employant le terme de "choquer" nous permet de mettre en lumière le sentiment visuel désagréable que peuvent représenter les écrans tactiles pour certains visiteurs. En 
regard, le verbe éclipser, signifiant "empêcher de paraitre, de briller, en brillant soimême davantage" exprime que les écrans tactiles peuvent être nuisibles pour les expôts et les cimaises explicatives. Un visiteur au musée Olympique s'est exprimé : "on ne se sent pas agressé mais invité à découvrir". Ce terme désigne directement l'aspect dérangeant, brutal que peuvent avoir ces écrans. Autrement dit, les écrans tactiles ne doivent apparemment pas s'imposer aux visiteurs au risque de tomber dans une dérive du "too much" qui importunerait. Ils doivent se proposer aux visiteurs en se fondant dans la scénographie.

L'esthétique est un autre point qui a retenu l'attention des visiteurs. Les musées sont tenus d'être beaux, de présenter une scénographie recherchée. Il est de mise pour eux de réussir à intégrer la technologie en proposant une approche contemporaine de leurs espaces. Il s'agit donc, pour les professionnels, de créer une scénographie qui séduit afin d'attirer les visiteurs dans leurs espaces. Pour y parvenir, les écrans tactiles semblent être une bonne solution car "ils participent à l'esthétique d'ensemble et donnent envie d'être touchés ". Particulièrement au CERN et au musée Olympique, les visiteurs ont trouvé que les écrans tactiles donnent une touche de modernité au musée et "diversifient la visite". Les visiteurs ont soulevé avec enthousiasme le bénéfice esthétique et moderniste de ces interfaces qui embellissent véritablement à leurs yeux les musées.

Des écrans aux contenus attractifs comme dans la partie consacrée à Olympie au musée Olympique de Lausanne.

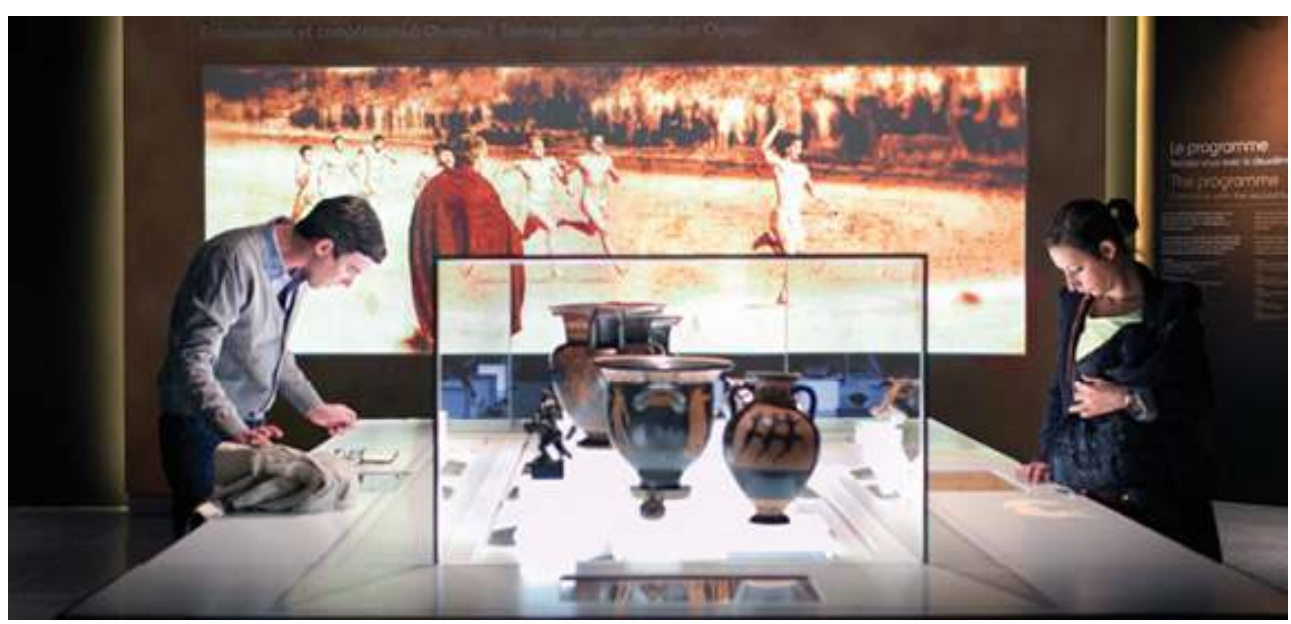

(c) Musée d'Aquitaine/Lysiane Gauthier

\section{Les informations délivrées par les contenus}

Le graphisme n'est pas un point sur lequel les visiteurs s'arrêtent spontanément. Ils emploient généralement des substantifs vagues pour le qualifier tels que "pas extraordinaire" pour le musée des Beaux-Arts de Bordeaux, "très bien fait, facilement lisible" pour l'Univers de Particules ou encore "moderne et beau, détaillé" au musée Olympique. Le contenu des écrans intéresse davantage les visiteurs. Nous avons remarqué qu'un contenu vide de sens, comme cela a été soulevé au musée des Beaux-Arts de Bordeaux avec des substantifs tels que "sans grand intérêt, décevant, trop limité", participe fortement à la dépréciation de l'écran. Il a d'ailleurs été demandé : "Les écrans apportent-ils plus que la classique fiche de salle?". À l'inverse, la diversité des informations que contiennent les 
bornes est saluée par les visiteurs. Ils estiment qu'il est "moins ennuyeux d'apprendre de cette manière". Il s'avère que les contenus doivent être attractifs pour justifier la présence de l'écran tactile, en plus ou en remplacement de la fiche de salle, par exemple.

31 Un point capital pour les visiteurs est le potentiel ludique, pour la jeunesse, de ces interfaces tactiles. Ce terme est revenu régulièrement dans les quatre musées. Le contenu des écrans tactiles est parfois directement estimé en termes de possibilité de jeux. N'estce pas là un sujet délicat? À la mode et grâce aux nouvelles méthodes d'enseignement scolaire, il est logique, pour les visiteurs, que les écrans tactiles dans les musées aient cette portée récréative. Aussi, devenu un mot passe-partout, le ludique est un terme que l'on emploie pour signifier tout ce qui est amusant, marrant. Les contenus muséaux ontils fondamentalement cette vocation? Non, ils ont pour but principal d'éduquer et de transmettre un réel contenu intellectuel, historique et patrimonial à buts pédagogiques. Nous avons donc constaté qu'un écran tactile, l'objet même, est rapidement considéré comme ludique. Du reste, des visiteurs se sont exclamés : "On apprend ou on joue ?", ce qui renforce cette ambiguité.

Aussi, la problématique de l'"edutainment" (Balloffet, Courvoisier et Lagier, pp. 4-16) reste très actuelle. Comment utilise-t-on ces écrans? Ici encore, une étude pourrait être réalisée sur l'intention que les visiteurs ont lorsqu'ils s'apprêtent à utiliser l'écran tactile. Cette problématique soulève un aspect essentiel du phénomène de l'intégration des écrans: ils sont une valeur ajoutée dans l'exposition et doivent défendre leur présence dans la scénographie grâce à leurs contenus.

\section{L'ergonomie}

33 L'usage des écrans tactiles doit être discuté pour comprendre la réussite ou non de ces dispositifs dans l'espace muséal; l'utilisation intuitive est une constante interrogation pour les musées. Le $100 \%$ de satisfaction quant à l'utilisation de ces dispositifs n'ayant jamais été atteint auprès des visiteurs de notre étude, force est de constater que certains utilisateurs ont rencontré quelques soucis fonctionnels. Tous les visiteurs ne savent pas comment aborder ces machines. Il a d'ailleurs été soulevé au musée d'Aquitaine qu' "il manque des encadrés expliquant le fonctionnement pour les personnes non habituées à l'utilisation de ces écrans". Ils ne comprendront pas, de ce fait, la possibilité de faire des zooms grâce à l'usage "Multi-touch", d'aller à gauche, à droite... Face à ces dispositifs, il y a un temps d'adaptation qui n'est pas négligeable. Certains visiteurs expriment que "c'est compliqué à manipuler si on ne connaît pas ce genre de surface". D'autres disent que "nous n'étions pas sûrs de faire juste". Ce cas n'est d'ailleurs pas isolé du Sud-Ouest de la France à l'Arc lémanique ! Une étude menée au musée de la Civilisation à Québec soulève la même problématique (Daignault et Cousson).

34 Au-delà de cette considération, la maniabilité de l'écran est un autre aspect important car elle pose parfois problème. Les visiteurs ont de la peine à saisir comment procéder selon qu'ils ont affaire à un écran plat posé horizontalement ou à des "drags balls" comme dans Univers de Particules. Il s'agit de relever que, même si les visiteurs sont rôdés à ce genre de technologie, ils peuvent rencontrer des difficultés. Certains "touchaient sans comprendre", alors que d'autres "ne comprenaient pas comment faire", s'il fallait "descendre ou monter". S'ajoutent à cela la sensibilité tactile des écrans et la réactivité de la commande exécutée. Certains de ces désagréments sont liés à une maniabilité élaborée, qui a peut-être un effet 
spectaculaire, mais qui est plus difficile à comprendre et qui fonctionne de manière bien plus sensible et délicate (Baccino, pp. 1-9).

Nous différencions bien le problème d'utilisation qui vient du visiteur et le souci de réactivité de la machine et la rapidité d'actualisation des mouvements du terminal. Effectivement, il ne s'agit pas toujours de la faute du visiteur. Les écrans tactiles demandent à être approchés le plus intuitivement possible et proposer une utilisation des plus faciles, basiques.

L'ergonomie des dispositifs tactiles peut parfois dérouter les visiteurs (Univers de Particules, CERN).

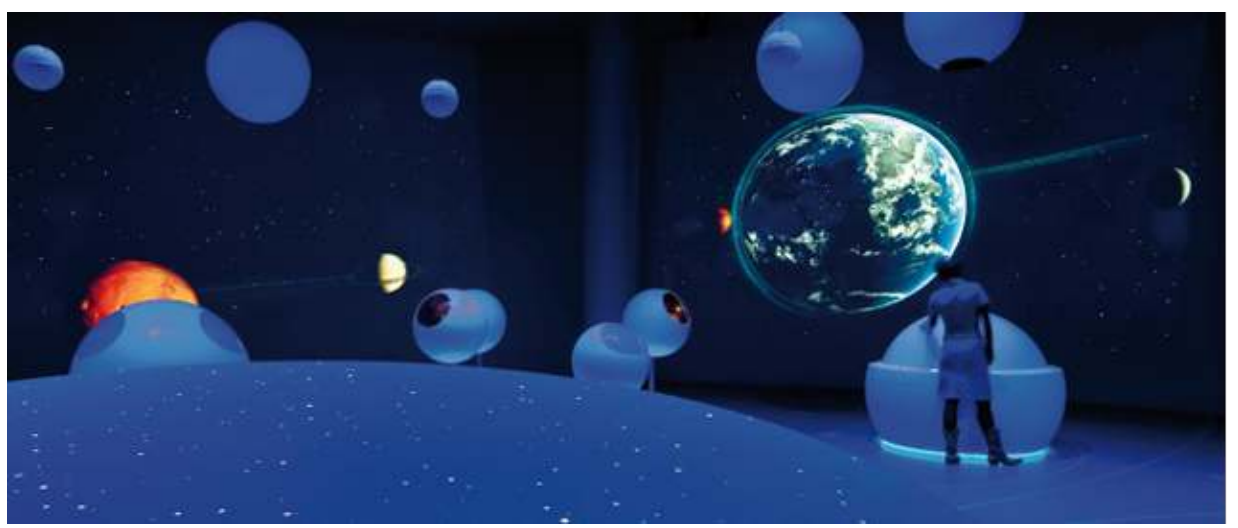

(c) CERN

\section{La pertinence d'écrans sur bornes fixes}

Les visiteurs estiment que les musées ont raison de s'ouvrir au monde numérique, les pourcentages d'acceptation ayant dépassé les $90 \%$ dans les quatre musées de notre étude, entre autres, pour des raisons pédagogiques ainsi que pour l'approche muséale. Après l'analyse des champs lexicaux issus des réponses linguistiques des visiteurs, attirer la jeunesse dans les musées semble être une évidence pour les visiteurs de notre étude: relevons les termes de "jeunes générations", "nouvelle génération", "les jeunes", "la jeunesse", "les petits et les ados"... Déjà habitués à l'utilisation de ces dispositifs, les jeunes visiteurs peuvent aborder le musée non pas en le voyant comme un endroit rébarbatif et ennuyeux, mais comme un espace culturel moderne faisant entièrement partie de la société actuelle. Car ces terminaux, d'après les visiteurs, contribuent à rendre la visite muséale plus enthousiasmante et leur donnent une nouvelle vision du musée.

Pour bon nombre de visiteurs, il s'agit même d'une habitude que de voir ces dispositifs dans tous les genres d'espaces. Il est alors normal de les insérer dans les musées. L'évolution technologique de la société induit donc, et logiquement, pour les visiteurs, que les outils technologiques soient insérés dans les musées avant que ceux-ci et leurs contenus ne soient perçus comme désuets. Le fait de les voir partout légitimise leur présence dans un lieu voué à l'extraordinaire. 
Des écrans qui ne doivent pas faire oublier aux visiteurs les œuvres et objets exposés (musée d'Aquitaine).

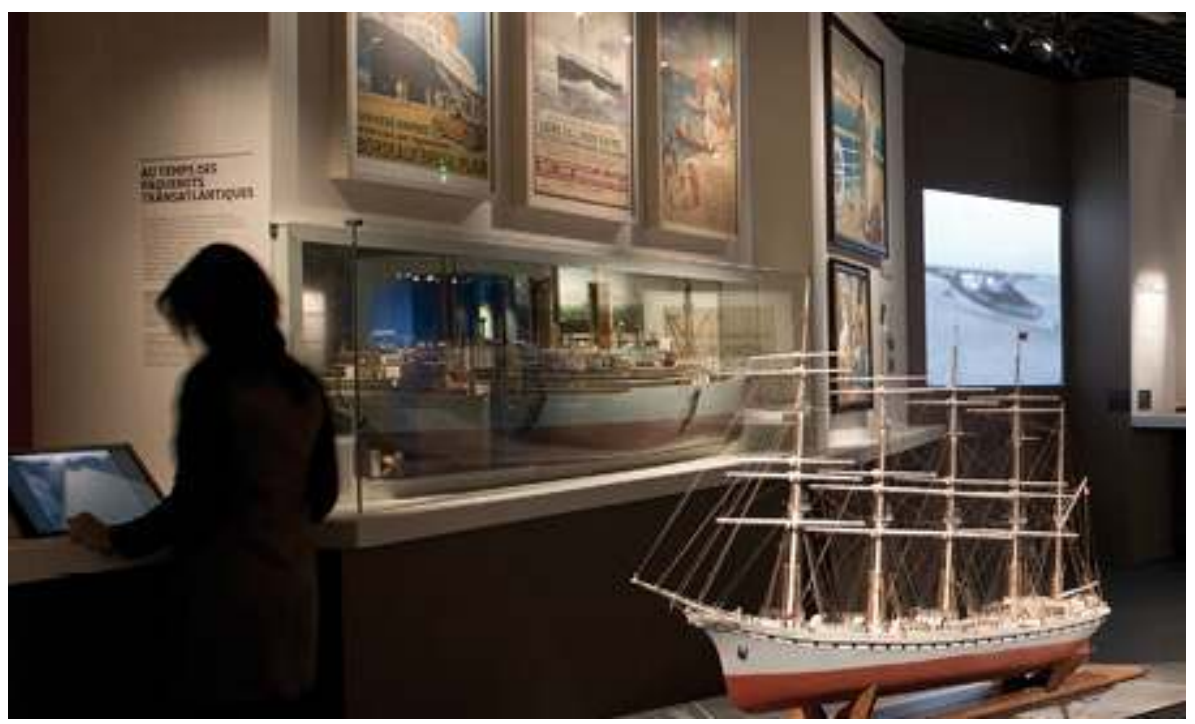

(c) Musée d'Aquitaine/Julie Guiroy consommation - il est devenu normal d'évoluer avec des outils tactiles, les visiteurs le soulèvent bien: "on vit avec des écrans, nous y sommes habitués". Leur intégration est devenue logique afin de communiquer sans créer de décalage avec la majorité des visiteurs. Le musée mute, s'adapte. Or, notre société changeant à vive allure impose une évolution fulgurante des nouvelles technologies qui se périment alors rapidement.

41 Nous voyons alors en ces objets le potentiel de diminution de la valeur d'usage. Ceci est dû au progrès technique, à l'apparition de nouveaux produits et à leur rapide obsolescence. Le musée, banalisant ces écrans tactiles, ne risque-t-il pas d'être à la mode quelque temps et de proposer des dispositifs technologiques rapidement obsolètes? Finalement, n'est-ce pas là compliquer la gestion de l'espace muséal et son bon 
fonctionnement que d'insérer des dispositifs interactifs tactiles? Ceci sous prétexte de mettre à disposition des contenus d'une nouvelle manière? Ces dispositifs sont-ils foncièrement nécessaires?

Cette étude exploratoire sur les écrans tactiles fixes ne représente qu'un temps de réflexion au cœur d'une évolution en marche accélérée. Aller au plus proche de visiteurs en leur transmettant un questionnaire permet d'émettre davantage que des hypothèses, des tendances indicatives afin de réfléchir sur l'expérience muséale revisitée grâce à l'ère numérique. Ainsi, les visiteurs étant en grande majorité conquis, la difficulté pour les musées ne sera plus uniquement de faire dialoguer les œuvres originales entre elles mais de faire dialoguer expôts et écrans tactiles, les deux dans une cohérence esthétique et un juste dosage.

Les auteurs tiennent à remercier Geneviève Dupuis-Sabron et Katia Kukawka (musée d'Aquitaine), Serge Fernandez (musée des Beaux-Arts de Bordeaux), Frédéric Jamolli, Anna Von Got et Christel Deshaie (musée Olympique de Lausanne), Bernard Pellequer (Microcosm et Univers de Particules).

\section{BIBLIOGRAPHIE}

Baccino, T. et al. L'usage des tablettes numériques : évaluation ergonomique. Colloque EcriTech'3, Nice, 5-6 avril 2012, pp. 1-9.

Balloffet, P., Courvoisier, F.-H. et Lagier, J. From Museum to Amusement Park: The Opportunities and Risks of Edutainment, International Journal of Arts Management, vol. 16, n², 2014, pp. 4-16.

Benjamin, W. L'œuvre d'art à l'époque de sa reproductibilité technique. Frankfurt am Main : Éditions Gallimard, 2000.

Bertrand, M. Les Technologies au musée : volonté d'afficher une modernité... déjà dépassée ? Comité de recherche, $\mathrm{n}^{\circ}$ 33, "Sociologie de la communication", université de Namur, 2010.

Coutaz, G. et Cosandier, J.-F. Avant-propos, in Patrimoine numérique, numérisation du patrimoine, Réseau Patrimoine, Association pour le patrimoine naturel et culturel du canton de Vaud, $\mathrm{n}^{\circ} 13$, IRL Plus, novembre 2012.

Daignault, L. et Cousson, C. Quand la technologie s'invite au musée, La Lettre de l'OCIM, n 137, septembre-octobre 2011, pp. 5-10.

Martin, Y.-A. Innovations numériques/révolution au musée ? Les Cahiers du Musée des Confluences, $\mathrm{n}^{\circ}$ 7, Innovation, juillet 2011, pp. 118-127.

Serres-Palson, E. Les nouveaux services communicationnels des musées. Médiation culturelle et NTIC en contexte muséal, in Médiation des cultures, actes des journées d'études du 26 et 27 mars 1999, Villeneuve d\&apos ;Ascq : université Charles-de-Gaulle-Lille III, Maison de la Recherche, 2000, pp. 37-47.

Torres, P., Treussard, L., Édouard-Baraud, R. et D’Anglejan, N. Impact des technologies numériques sur le monde de la culture : bilan de nos 10 premières années au 21e siècle.

Éditions l'Atelier Études et Conseil pour le Forum d'Avignon, 2010. 
Vol, A. Tisser des trames de pertinence entre musées, nouvelles technologies et publics, Publics et Musées, $\mathrm{n}^{\circ}$ 13, 1998, pp. 67-87.

Vidal, G. Interactivité et médiation dans l'usage des multimédias de musées, Communication et langages, $\mathrm{n}^{\circ} 137,2003$, pp. 63-76.

\section{NOTES}

1. A cet effet, il est nécessaire de mentionner que le Musée des Beaux-Arts de Bordeaux a rapidement renoncé à ce projet, faute de succès et de budget, au détriment d'une table Multitouch. Nous avons décidé de conserver l'étude qui a été effectuée car elle est représentative pour notre analyse.

\section{RÉSUMÉS}

L'étude présentée ici met en évidence les nouvelles possibilités et appréhensions qu'ont les visiteurs lorsqu'ils pénètrent dans un espace muséal qui propose, au sein de ses expositions, des interfaces tactiles fixes : elle pointe les implications de ce phénomène qui se généralise dans les institutions muséales.

\section{INDEX}

Mots-clés : écran tactile, musée, visiteur

\section{AUTEURS}

\section{CHARLOTTE STEINER}

diplômée d'un Master en études muséales, est stagiaire au département des objets au musée Historique de Lausanne

charlotte.steiner@hotmail.com

\section{FRANÇOIS H. COURVOISIER}

professeur HES, doyen de l\&apos ;Institut du Marketing Horloger à la Haute école de gestion Arc, Espace de 1\&apos ;Europe 21 à Neuchâtel francois.courvoisier@he-arc.ch fr 